\title{
A Generalization of Uniformly Extremely Convex Banach Spaces
}

\author{
Suyalatu Wulede, ${ }^{1}$ Wurichaihu Bai, ${ }^{1}$ and Wurina Bao' \\ ${ }^{1}$ College of Mathematics Science, Inner Mongolia Normal University, Hohhot 010022, China \\ ${ }^{2}$ Xilingol Vocational College, Xilinhot 026000, China
}

Correspondence should be addressed to Suyalatu Wulede; suyila520@163.com

Received 19 January 2016; Accepted 16 March 2016

Academic Editor: Gianluca Vinti

Copyright (C) 2016 Suyalatu Wulede et al. This is an open access article distributed under the Creative Commons Attribution License, which permits unrestricted use, distribution, and reproduction in any medium, provided the original work is properly cited.

\begin{abstract}
We discuss a new class of Banach spaces which are the generalization of uniformly extremely convex spaces introduced by Wulede and Ha. We prove that the new class of Banach spaces lies strictly between either the classes of $k$-uniformly rotund spaces and $k$-strongly convex spaces or classes of fully $k$-convex spaces and $k$-strongly convex spaces and has no inclusive relation with the class of locally $k$-uniformly convex spaces. We obtain in addition some characterizations and properties of this new class of Banach spaces. In particular, our results contain the main results of Wulede and Ha.
\end{abstract}

\section{Introduction}

Different uniformly convex spaces have been defined between the uniformly convex spaces [1] and the reflexivity of the Banach spaces [2-6]. In the previous paper [7] we introduce a new class of this type, namely, uniformly extremely convex spaces. This new class of Banach spaces lies strictly between either the classes of uniformly convex spaces and strongly convex spaces or the classes of fully $k$-convex spaces and strongly convex spaces.

Here we consider another new class of this type, namely, $k$-uniformly extremely convex spaces, as a generalization of uniformly extremely convex spaces and discuss its relation to the drop property, the $k$-uniformly rotund spaces, the full $k$-convex spaces, the $k$-strongly convex spaces, the nearly uniformly convex spaces, and $k$-nearly uniformly convex spaces. We also give some characterizations of $k$-uniformly extremely convex spaces and find that this new class of Banach spaces has the following features:

(1) 1-uniformly extremely convex spaces (indeed lower case) coincide with uniformly extremely convex spaces;

(2) $k$-uniformly extremely convex spaces possess the drop linebreak property;
(3) $k$-uniformly extremely convex spaces are $(k+1)$ uniformly extremely convex spaces, but the converse implication is not true.

Throughout this paper $X$ denotes an infinite-dimensional real Banach space with the norm $\|\cdot\|$. The symbol $X^{*}$ denotes the dual of the space $X . U(X)$ and $S(X)$ denote the closed unit ball and the unit sphere of $X$, respectively. The symbol $S\left(X^{*}\right)$ denotes the unit sphere of $X^{*}$. The symbol $\sigma\left(X, X^{*}\right)$ denotes the weak topology of $X$.

Let $x_{1}, x_{2}, \ldots, x_{k+1}$ be norm-1 elements in Banach spaces $X$. The $k$-dimensional volume enclosed by $x_{1}, x_{2}, \ldots, x_{k+1}$ is given by

$$
\begin{aligned}
& V\left(x_{1}, x_{2}, \ldots, x_{k+1}\right) \\
& =\sup \left\{\begin{array}{cccc}
1 & 1 & \ldots & 1 \\
f_{1}\left(x_{1}\right) & f_{1}\left(x_{2}\right) & \cdots & f_{1}\left(x_{k+1}\right) \\
\vdots & \vdots & \ddots & \vdots \\
f_{k}\left(x_{1}\right) & f_{k}\left(x_{2}\right) & \cdots & f_{k}\left(x_{k+1}\right)
\end{array} \mid: f_{1}, \ldots, f_{k}\right. \\
& \left.\quad \in S\left(X^{*}\right)\right\} .
\end{aligned}
$$


Here, and throughout the sequel, the symbol $|\cdot|$ denotes the determinant.

Sullivan [6] has introduced the $k$-uniformly rotund ( $k \mathrm{UR}$ ) spaces and locally $k$-uniformly rotund (L $k \mathrm{UR}$ ) spaces. Fan and Glicksberg [2] have introduced the fully $k$-convex $(k \mathrm{R})$ Banach spaces. It is well known that $k \mathrm{UR}$ and $k \mathrm{R}$ spaces imply reflexivity. About $k \mathrm{UR}$ and $k \mathrm{R}$ spaces, we have the following chain of implications $[2,6,8]$ :

$$
\begin{aligned}
\mathrm{UR} & =1 \mathrm{UR} \Longrightarrow \cdots \Longrightarrow k \mathrm{UR} \Longrightarrow(k+1) \mathrm{UR} ; \\
2 \mathrm{R} & \Longrightarrow \cdots \Longrightarrow k \mathrm{R} \Longrightarrow(k+1) \mathrm{R} ; \\
\mathrm{LUR} & =\mathrm{L} 1 \mathrm{UR} \Longrightarrow \cdots \Longrightarrow \mathrm{L} k \mathrm{UR} \Longrightarrow L(k+1) \mathrm{UR} .
\end{aligned}
$$

A Banach space $X$ is said to be a $k U R$ space $(k \geq 1)[6]$ if, for any $\epsilon>0$, there exists a $\delta(\epsilon)>0$ such that, for all norm-1 elements $x_{1}, x_{2}, \ldots, x_{k+1}$ and $\left\|x_{1}+x_{2}+\cdots+x_{k+1}\right\|>(k+1)-\delta$, we have $V\left(x_{1}, x_{2}, \ldots, x_{k+1}\right)<\epsilon$.

A Banach space $X$ is said to be a $k \mathrm{R}$ space $(k \geq 2)$ [2] if, for any sequence $\left\{x_{n}\right\}$ in $X$ such that $\lim _{n_{1}, \ldots, n_{k} \rightarrow \infty}(1 / k) \| x_{n_{1}}+$ $x_{n_{2}}+\cdots+x_{n_{k}} \|=1$, then $\left\{x_{n}\right\}$ is a Cauchy sequence in $X$.

A point $x_{0} \in S(X)$ is said to be a denting point of $U(X)$ [8] if $x_{0} \notin \overline{\mathrm{co}}\left(M\left(x_{0}, \epsilon\right)\right)$ for all $\epsilon>0$, where $M\left(x_{0}, \epsilon\right)=\{y$ : $\left.y \in U(X),\left\|y-x_{0}\right\| \geq \epsilon\right\}$.

Huff [3] has introduced the nearly uniformly convex (NUC) spaces as a generalization of uniformly convex Banach spaces and showed that the NUC spaces are equivalent to reflexive spaces possessing the uniform Kadec-Klee property. The local version of NUC spaces, namely, locally nearly uniformly convex (LNUC), was studied by Kutzarova and Lin [9]. Kutzarova [4] introduced the $k$-nearly uniformly convex ( $k$ NUC) spaces as a generalization of nearly uniformly convex Banach spaces. In $[4,9]$, it is pointed out that NUC $\Rightarrow$ LNUC and $k$ NUC $\Rightarrow$ NUC for every $k \geq 2$.

A Banach space $X$ is said to be a NUC [3] space if, for any $\epsilon>0$, there exists a $\delta(\epsilon)>0$ such that, for any sequence $\left\{x_{n}\right\} \subset U(X), \operatorname{sep}\left(x_{n}\right)>\epsilon$, we have $\operatorname{co}\left(\left\{x_{n}\right\}\right) \cap(1-\delta) U(X) \neq \emptyset$, where $\operatorname{sep}\left(x_{n}\right)=\inf \left\{\left\|x_{n}-x_{m}\right\|: n \neq m\right\}$ and $\operatorname{co}\left(\left\{x_{n}\right\}\right)$ means the convex hull of $\left\{x_{n}\right\}$.

A Banach space $X$ is said to be a LNUC [9] space if, for any norm-1 element $x$ and $\epsilon>0$, there exists a $\delta=\delta(\epsilon, x)>0$ such that, for any sequence $\left\{x_{n}\right\} \subset U(X), \operatorname{sep}\left(x_{n}\right)>\epsilon$, we have $\operatorname{co}\left(\{x\} \cup\left\{x_{n}\right\}\right) \cap(1-\delta) U(X) \neq \emptyset$, where $\operatorname{co}\left(\{x\} \cup\left\{x_{n}\right\}\right)$ means the convex hull of $\{x\}$ and $\left\{x_{n}\right\}$.

A Banach space $X$ is said to be a $k$ NUC [4] space, if for any $\epsilon>0$ there exists a $0<\delta(\epsilon)<1$ such that, for any sequence $\left\{x_{n}\right\} \subset U(X), \operatorname{sep}\left(x_{n}\right)>\epsilon$, there are indices $\left\{n_{i}\right\}$ and scalars $\lambda_{i} \geq 0, i=1,2, \ldots, k$, with $\sum_{i=1}^{k} \lambda_{i}=1$ so that $\left\|\sum_{i=1}^{k} \lambda_{i} x_{n_{i}}\right\| \leq$ $1-\delta$.

Singer [10] has introduced the $k$-strictly convex spaces. It is well known that $k$-strictly convex spaces are $(k+1)$-strictly convex spaces; 1 -strictly convex spaces (indeed lower case) coincide with strictly convex spaces; $k \mathrm{R}$ spaces are $k$-strictly convex spaces and have the drop property. Wu and $\mathrm{Li}$ [11] have introduced the strongly convex spaces. Wulede and $\mathrm{Wu}$ [12] introduced the $k$-strongly convex spaces as a generalization of strongly convex Banach spaces and gave an equivalent definition of $k$-strongly convex spaces (see Theorem 5 in [13]).
It is well known that $k$-strongly convex spaces are $k$-strictly convex spaces; 1-strongly convex spaces (indeed lower case) coincide with strongly convex spaces; $k$-strongly convex spaces are $(k+1)$-strongly convex spaces, but the converse implication is not true.

A Banach space $X$ is said to be a $k$-strictly convex space [10] if, for all norm-1 elements $x_{1}, x_{2}, \ldots, x_{k+1}$ such that $\left\|\sum_{i=1}^{k+1} x_{i}\right\|=k+1$, then $x_{1}, x_{2}, \ldots, x_{k+1}$ are linearly dependent.

A Banach space $X$ is said to be a strongly convex space [11] if, for any $x \in S(X),\left\{x_{n}\right\} \subset S(X)$ and for a certain functional $f \in S_{x}$ such that $f\left(x_{n}\right) \rightarrow 1(n \rightarrow \infty)$, then $\left\|x_{n}-x\right\| \rightarrow$ $0(n \rightarrow \infty)$, where $S_{x}=\left\{f: f \in S\left(X^{*}\right), f(x)=1\right\}$.

A Banach space $X$ is said to be a $k$-strongly convex space [12] if, for any norm-1 element $x, \epsilon>0$ and for any functional $f \in S_{x}$, there is a $\delta(x, f, \epsilon)>0$ such that, for all norm-1 elements $x_{1}, \ldots, x_{k}$ and $f\left(x+x_{1}+\cdots+x_{k}\right)>(k+1)-\delta$, we have $V\left(x, x_{1}, \ldots, x_{k}\right)<\epsilon$.

Rolewicz [14] has defined the norm $\|\cdot\|$ to have the drop property, if for every closed set $C \subset X$ disjoint from $U(X)$ there exists $x \in C$ such that $D(x, U(X)) \cap C=\{x\}$, where the set $D(x, U(X))$, the convex hull of $x$ and $U(X)$, is called the drop generated by $x \notin U(X)$.

Lemma 1 (Kadec-Klee property). If any $x \in S(X),\left\{x_{n}\right\} \subset$ $S(X)$ such that $x_{n} \stackrel{w}{\rightarrow} x, n \rightarrow \infty$, and $\left\|x_{n}\right\| \rightarrow\|x\|, n \rightarrow \infty$, then $\left\|x_{n}-x\right\| \rightarrow 0, n \rightarrow \infty$, where $x_{n} \stackrel{w}{\rightarrow} x, n \rightarrow \infty$, means that $f\left(x_{n}\right) \rightarrow f(x), n \rightarrow \infty$, for all $f \in X^{*}$.

Lemma 2 (Montesinos [15]). Let $X$ be a Banach space. Then $X$ has the drop property if and only if $X$ is reflexive and has the Kadec-Klee property.

Lemma 3 (Nan and Wang [16]). $X$ is $k$-strictly convex space if and only if, for any $f \in S\left(X^{*}\right)$, one has $\operatorname{dim} A_{f} \leq k$, where $A_{f}=\{x: x \in S(X), f(x)=1\}$.

Lemma 4 (Wulede and Wu [12], Zhang and Fang [17]). Let X be a Banach space.

(i) If $X$ is $k$-strongly convex, then $X$ is $k$-strictly convex and has the Kadec-Klee property.

(ii) If $X$ is reflexive, $k$-strictly convex and has the KadecKlee property, then $X$ is $k$-strongly convex.

(iii) If $X$ is $k$-strongly convex, $\left\{x_{n}\right\} \subset U(X), f \in S\left(X^{*}\right)$, and $f\left(x_{n}\right) \rightarrow 1, n \rightarrow \infty$, then $\operatorname{dist}\left(x_{n}, A_{f}\right) \rightarrow 0, n \rightarrow \infty$.

Lemma 5 (Zhang and Fang [17]). $X$ is $k$-strongly convex if and only if, for any $x \in S(X)$ and $f \in S_{x}, \epsilon>0$, there exist $\delta>0$ and a compact set $C \subset X$ with $\operatorname{dim} C \leq k$ such that $F(f, \delta) \subset$ $\{x: x \in X, d(x, C)<\epsilon\}$, where the set $F(f, \delta)=\{x: x \in$ $U(X), f(x) \geq 1-\delta\}$ is the slice generated by $f$ and $\delta$.

\section{2. $k$-Uniformly Extremely Convex Spaces and Drop Property}

Definition 6 (see [7]). A Banach space $X$ is said to be a uniformly extremely convex space if, for any sequences 
$\left\{x_{n}\right\},\left\{y_{n}\right\}$ consisting of elements of norm-1 and for a certain functional $f$ of norm-1, $\lim _{n \rightarrow \infty} f\left(x_{n}\right)=\lim _{n \rightarrow \infty} f\left(y_{n}\right)=1$ holds; then $\lim _{n \rightarrow \infty}\left\|x_{n}-y_{n}\right\|=0$.

On the base of uniformly extremely convex spaces, now we introduce the notion of $k$-uniformly extremely convex spaces as a generalization of uniformly extremely convex spaces.

Definition 7. A Banach space $X$ is said to be a $k$-uniformly extremely convex space if, for any sequences $\left\{x_{n}^{(1)}\right\}, \ldots,\left\{x_{n}^{(k+1)}\right\}$ consisting of elements of norm- 1 and for a certain functional $f$ of norm-1, $\lim _{n \rightarrow \infty} f\left(x_{n}^{(1)}\right)=\cdots=\lim _{n \rightarrow \infty} f\left(x_{n}^{(k+1)}\right)=1$ holds; then $\lim _{n \rightarrow \infty} V\left(x_{n}^{(1)}, \ldots, x_{n}^{(k+1)}\right)=0$.

We give first a simple result which shows that the notion of $k$-uniformly extremely convex space is "coherent."

Theorem 8. If $X$ is $k$-uniformly extremely convex space, then $X$ is $(k+1)$-uniformly extremely convex space.

Proof. If, for any sequences $\left\{x_{n}^{(1)}\right\}, \ldots,\left\{x_{n}^{(k+2)}\right\}$ consisting of elements of norm- 1 and for a certain functional $f$ of norm-1, $\lim _{n \rightarrow \infty} f\left(x_{n}^{(1)}\right)=\cdots=\lim _{n \rightarrow \infty} f\left(x_{n}^{(k+2)}\right)=1$ holds, then, for all $1 \leq j \leq k+2$, we have $\lim _{n \rightarrow \infty} V\left(x_{n}^{(1)}, \ldots, x_{n}^{(j-1)}, x_{n}^{(j+1)}, \ldots\right.$, $\left.x_{n}^{(k+2)}\right)=0$ by the assumption that $X$ is $k$-uniformly extremely convex space. Furthermore, by the properties of determinant we have

$$
\begin{aligned}
& \lim _{n \rightarrow \infty} V\left(x_{n}^{(1)}, \ldots, x_{n}^{(k+2)}\right) \\
& \quad \leq \lim _{n \rightarrow \infty} \sum_{j=2}^{k+1} V\left(x_{n}^{(1)}, \ldots, x_{n}^{(j-1)}, x_{n}^{(j+1)}, \ldots, x_{n}^{(k+2)}\right)=0 ;
\end{aligned}
$$

this shows that $X$ is $(k+1)$-uniformly extremely convex space.

Now we give a simple but useful lemma. By using this lemma we can prove that any $k$-uniformly extremely convex space has the drop property. And the fact that $k$-uniformly extremely convex spaces include $k$-strongly convex spaces can be easily found. We also show that 1-uniformly extremely convex spaces coincide with uniformly extremely convex spaces by using this lemma.

Lemma 9. $X$ is k-uniformly extremely convex if and only if, for any $\epsilon>0, f \in S\left(X^{*}\right)$, there exists a $\delta(\epsilon)>0$ such that, for all norm-1 elements $x_{1}, \ldots, x_{k+1}$ and $f\left(\sum_{i=1}^{k+1} x_{i}\right)>(k+1)-\delta$, one has $V\left(x_{1}, \ldots, x_{k+1}\right)<\epsilon$.

Proof.

Necessity. Suppose the contrary. Then there exist $\epsilon_{0}>0, f_{0} \in$ $S\left(X^{*}\right)$ and $\left\{x_{i}\right\}_{i=1}^{k+1} \subset S(X)$ such that, for any $\delta=1 / n, n \in N$, we have $f_{0}\left(\sum_{i=1}^{k+1} x_{i}\right)>(k+1)-1 / n$, but $V\left(x_{1}, \ldots, x_{k+1}\right) \geq \epsilon_{0}$. Take $x_{n}^{(i)}=x_{i}(i=1, \ldots, k+1)$; then $\left\{x_{n}^{(i)}\right\}_{i=1}^{k+1} \subset S(X)$ and $k+1-1 / n<f_{0}\left(\sum_{i=1}^{k+1} x_{n}^{(i)}\right) \leq k+1$. It follows that $\lim _{n \rightarrow \infty} f_{0}\left(x_{n}^{(i)}\right)=1$. On the other hand, by the definition of the $k$-uniformly extremely convex space, we have $V\left(x_{1}, \ldots, x_{k+1}\right) \rightarrow 0$; this contradicts the statement that $V\left(x_{1}, \ldots, x_{k+1}\right) \geq \epsilon_{0}$.

Sufficiency. If, for any sequences $\left\{x_{n}^{(1)}\right\}, \ldots,\left\{x_{n}^{(k+1)}\right\}$ consisting of elements of norm- 1 and for a certain functional $f$ of norm1, $\lim _{n \rightarrow \infty} f\left(x_{n}^{(1)}\right)=\cdots=\lim _{n \rightarrow \infty} f\left(x_{n}^{(k+1)}\right)=1$ holds, then $\lim _{n \rightarrow \infty} f\left(\sum_{i=1}^{k+1} x_{n}^{(i)}\right)=k+1$. Therefore, for any $\delta>0$, there exists an integer $N_{0} \in N$ such that, for all $n \geq N_{0}$, inequality $f\left(\sum_{i=1}^{k+1} x_{n}^{(i)}\right)>(k+1)-\delta$ holds. For any $\epsilon>0$, by the conditions given in Lemma 9, we have $V\left(x_{n}^{(1)}, \ldots, x_{n}^{(k+1)}\right)<\epsilon$; this means that $\lim _{n \rightarrow \infty} V\left(x_{n}^{(1)}, \ldots, x_{n}^{(k+1)}\right)=0$.

Remark 10. 1-uniformly extremely convex space (indeed lower case) coincides with uniformly extremely convex space.

In fact, by Lemma 9 we know that $X$ is 1-uniformly extremely convex space if and only if, for any $\epsilon>0, f \epsilon$ $S\left(X^{*}\right)$, there exists a $\delta(\epsilon, f)>0$ such that, for any norm-1 elements $x, y$ and $f(x+y)>2-\delta$, we have

$$
\begin{aligned}
V(x, y) & =\sup \left\{\left|\begin{array}{cc}
1 & 1 \\
f(x) & f(y)
\end{array}\right|: f \in S\left(X^{*}\right)\right\} \\
& =\sup _{f \in S\left(X^{*}\right)}|f(x)-f(y)|=\|x-y\|<\epsilon,
\end{aligned}
$$

and also if and only if $X$ is uniformly extremely convex space.

Theorem 11. $X$ is $k$-uniformly extremely convex space if and only if $X$ is $k$-strictly convex space and has the drop property.

Proof.

Necessity. Suppose that $X$ is $k$-uniformly extremely convex space; by the definition of $k$-strongly convex space and a condition which characterizes $k$-uniformly extremely convex space in Lemma 9, it is easy to see that $X$ is $k$-strongly convex space. From Lemma 4(i), we know that $X$ is $k$-strictly convex space and has the Kadec-Klee property.

Now we are going to prove that $X$ has the drop property. In fact, from Lemma 2, it is sufficient to prove that $X$ is reflexive. Suppose that $X$ is not reflexive. Using the wellknown James' theorem, for each $0<\epsilon<1$, we can choose $0<\theta<1$ so that $\theta>1-\delta(\epsilon) /(k+1)$ and $\theta^{k}>\epsilon$, and $\left\{x_{1}\right\}, \ldots,\left\{x_{k+1}\right\} \subset S(X),\left\{x_{1}^{*}\right\}, \ldots,\left\{x_{k+1}^{*}\right\} \subset S\left(X^{*}\right)$ so that

$$
x_{j}^{*}\left(x_{i}\right)= \begin{cases}\theta & \text { if } j \leq i \\ 0 & \text { if } j>i .\end{cases}
$$

Here $\delta(\epsilon)$ is the function required in Lemma 9.

Now we have that

$$
x_{1}^{*}\left(x_{1}+x_{2}+\cdots+x_{k+1}\right)=(k+1) \theta>(k+1)-\delta(\epsilon) .
$$


On the other hand it is easy to check that

$$
\begin{aligned}
& V\left(x_{1}, \ldots, x_{k+1}\right) \\
& \geq\left|\begin{array}{cccc}
1 & 1 & \ldots & 1 \\
x_{2}^{*}\left(x_{1}\right) & x_{2}^{*}\left(x_{2}\right) & \cdots & x_{2}^{*}\left(x_{k+1}\right) \\
\vdots & \vdots & \ddots & \vdots \\
x_{k+1}^{*}\left(x_{1}\right) & x_{k+1}^{*}\left(x_{2}\right) & \cdots & x_{k+1}^{*}\left(x_{k+1}\right)
\end{array}\right|=\theta^{k}>\epsilon,
\end{aligned}
$$

which gives the required contradiction.

Sufficiency. From the assumption that $X$ is $k$-strictly convex space and has the drop property, we can deduce, by Lemma 4 (ii), that $X$ is $k$-strongly convex space and reflexive. Observing the definition of $k$-strongly convex space and a condition which characterizes $k$-uniformly extremely convex space in Lemma 9, by the reflexivity of $X$, it is easy to see that $X$ is $k$-uniformly extremely convex space.

Corollary 12 (see [7]). $X$ is uniformly extremely convex space if and only if $X$ is strictly convex space and has the drop property.

Noticing the procedure of proving Theorem 11 we can deduce the following.

Corollary 13. If $X$ is $k$-uniformly extremely convex space, then $X$ is k-strongly convex space.

Now we are going to show that the converse to Corollary 13 is not true. In [12], it is proved that LkUR spaces are $k$-strongly convex spaces. In general, L $k U R$ spaces need not be reflexive since L1UR is just the usual definition of LUR space $[18,19]$. It follows that there exists a $k$-strongly convex space $X$ which is not reflexive. Hence $X$ is not a $k$-uniformly extremely convex space since $X$ is not reflexive.

Corollary 14. $X$ is $k$-uniformly extremely convex space if and only if $X$ is reflexive and, for any $x \in S(X)$ and $f \in S_{x}, \epsilon>0$, there exist $\delta>0$ and a compact set $C \subset X$ with $\operatorname{dim} C \leq k$ such that $F(f, \delta) \subset\{x: x \in X, d(x, C)<\epsilon\}$, where the set $F(f, \delta)=\{x: x \in U(X), f(x) \geq 1-\delta\}$ is the slice generated by $f$ and $\delta$.

Proof. It is immediate from Corollary 13, Theorem 11, and Lemmas 2, 4, and 5.

Theorem 15. $X$ is $k$-uniformly extremely convex space if and only if $X$ is reflexive and, for any $f \in S\left(X^{*}\right)$, one has $\operatorname{dim} A_{f} \leq$ $k, A_{f} \cap \overline{\mathrm{co}}\left(U(X) \backslash V_{A_{f}}\right)=\emptyset$, where the set $V_{A_{f}}$, which includes set $A_{f}$, is arbitrary open set with regard to norm topology $(X,\|\cdot\|)$.

\section{Proof.}

Necessity. Suppose that $X$ is $k$-uniformly extremely convex space; then by Theorem 11 we know that $X$ is $k$-strictly convex space and reflexive. For any $f \in S\left(X^{*}\right)$, by the reflexivity of $X$, there exists $x \in S(X)$ such that $f(x)=1$; hence $x \in A_{f}$.
Combining the fact that $X$ is $k$-strictly convex space with Lemma 3 we can deduce that $\operatorname{dim} A_{f} \leq k$.

Now we are going to prove the equality $A_{f} \cap \overline{\mathrm{co}}(U(X) \backslash$ $\left.V_{A_{f}}\right)=\emptyset$.

Firstly, we will prove that, for any $z \notin V_{A_{f}}$ and every open set $V_{A_{f}}$ (where $V_{A_{f}} \supset A_{f}$ ) with regard to norm topology $(X,\|\cdot\|)$, there exists a scalar $r>0$ such that $\operatorname{dist}\left(z, A_{f}\right) \geq r$.

Noticing that $A_{f}$ is compact set in $X$, for any $z \notin V_{A_{f}}$, we can deduce that there exists $x \in A_{f}$ such that $\|x-z\|=$ $\operatorname{dist}\left(z, A_{f}\right)=r_{z}$. Now we claim that there exists minimum value of $r_{z}$ denoted by $r$, such that $\operatorname{dist}\left(z, A_{f}\right) \geq r$ for any $z \notin V_{A_{f}}$. In fact, if $r_{z}$ does not have minimum value, then $1 / n$ is impossible to be minimum value for any integer $n$. Hence, there exist $z_{n} \notin V_{A_{f}}$ and $x_{n} \in A_{f}$ such that $\| x_{n}-$ $z_{n} \|=\operatorname{dist}\left(z_{n}, A_{f}\right)<1 / n$. Since $A_{f}$ is compact, the above sequence $\left\{x_{n}\right\}$ has the convergent subsequence; without loss of generality and letting the convergent subsequence be $\left\{x_{n}\right\}$ itself, then $x_{n} \rightarrow x_{0}, x_{0} \in A_{f}$. Noticing that $\left\|x_{n}-z_{n}\right\|=$ $\operatorname{dist}\left(z_{n}, A_{f}\right)<1 / n$, we can deduce that $z_{n} \rightarrow x_{0}, x_{0} \in A_{f} \subset$ $V_{A_{f}}$.

On the other hand, combining the fact that $X \backslash V_{A_{f}}$ is closed set with $z_{n} \in X \backslash V_{A_{f}}, z_{n} \rightarrow x_{0}$, we can deduce that $x_{0} \in X \backslash V_{A_{f}}$. This contradicts $x_{0} \subset V_{A_{f}}$.

Secondly, we will prove that for $V_{A_{f}}$ there exists a scalar $m>0$ such that the inequality $f(x)>f(y)+m$ holds for all $x \in A_{f}$ and $y \in U(X) \backslash V_{A_{f}}$.

If the above inequality is not true, then there exists $y_{n} \in$ $U(X) \backslash V_{A_{f}}$ such that $f\left(y_{n}\right) \rightarrow f(x)=1, n \rightarrow \infty$. By the condition given in Theorem 15, Corollary 13, and Lemma 4(iii), we have $\operatorname{dist}\left(y_{n}, A_{f}\right) \rightarrow 0, n \rightarrow \infty$. On the other hand, by $y_{n} \in U(X) \backslash V_{A_{f}}$, we can deduce that $\operatorname{dist}\left(y_{n}, A_{f}\right) \nrightarrow 0, n \rightarrow \infty$; this contradicts the statement that $\operatorname{dist}\left(y_{n}, A_{f}\right) \rightarrow 0, n \rightarrow \infty$. Hence we have

$$
\begin{aligned}
f(x)-m & \geq \sup \left\{f(y): y \in U(X) \backslash V_{A_{f}}\right\} \\
& =\sup \left\{f(y): y \in \overline{\mathrm{co}}\left(U(X) \backslash V_{A_{f}}\right)\right\} ;
\end{aligned}
$$

this shows that $x \notin \overline{\mathrm{co}}\left(U(X) \backslash V_{A_{f}}\right)$. By the arbitrary of $x \in$ $A_{f}$, we can deduce that $A_{f} \cap \overline{\mathrm{co}}\left(U(X) \backslash V_{A_{f}}\right)=\emptyset$.

Sufficiency. By Lemmas 2 and 3, Theorem 11, and the condition given in Theorem 15, only we need to prove that $X$ has the Kadec-Klee property. Let $x \in S(X),\left\{x_{n}\right\}_{n=1}^{\infty} \subset S(X)$, and $x_{n} \stackrel{w}{\rightarrow} x, n \rightarrow \infty$. By the well-known James' theorem, there exists $f \in S\left(X^{*}\right)$ such that $f(x)=1$; it follows that $x \in A_{f}$.

Case 1. If $\left\{x_{n}\right\}_{n=1}^{\infty} \cap A_{f}=\emptyset$, then $\left\{x_{n}\right\}_{n=1}^{\infty}$ is relatively compact. Otherwise, every point of $A_{f}$ is not accumulation point of $\left\{x_{n}\right\}_{n=1}^{\infty}$. Hence, for any $x \in A_{f}$ there exists $\epsilon_{0}>0$ such that $\left\{y \in X:\|y-x\|<\epsilon_{0}\right\}$ does not contain any point of $\left\{x_{n}\right\}_{n=1}^{\infty}$. We construct an open set $V_{A_{f}}=\bigcup_{x \in A_{f}}\{y \in X$ : $\left.\|y-x\|<\epsilon_{0}\right\}$ with regard to norm topology $(X,\|\cdot\|)$; then $A_{f} \subset V_{A_{f}}$ and $V_{A_{f}} \cap\left\{x_{n}\right\}_{n=1}^{\infty}=\emptyset$. Since $\overline{\operatorname{co}}\left(U(X) \backslash V_{A_{f}}\right)$ is bounded closed convex set with regard to norm topology 
$(X,\|\cdot\|), \overline{\mathrm{co}}^{w}\left(U(X) \backslash V_{A_{f}}\right)=\overline{\mathrm{co}}\left(U(X) \backslash V_{A_{f}}\right)$. Noticing that $\overline{\mathrm{co}}^{w}\left(U(X) \backslash V_{A_{f}}\right)$ is bounded set with regard to weak topology $\sigma\left(X, X^{*}\right)$, we know that $\overline{\mathrm{co}}^{w}\left(U(X) \backslash V_{A_{f}}\right)$ is compact set with regard to weak topology $\sigma\left(X, X^{*}\right)$. Hence there is a function $g \in X^{*}$ which separates $A_{f}$ and $\overline{\operatorname{co}}\left(U(X) \backslash V_{A_{f}}\right)$; that is, there is a scalar $l>0$ such that $g\left(A_{f}\right)-l>\sup g(\overline{\mathrm{co}}(U(X) \backslash$ $\left.V_{A_{f}}\right)$ ). Evidently, $\left\{x_{n}\right\}_{n=1}^{\infty} \subset \overline{\mathrm{Co}}\left(U(X) \backslash V_{A_{f}}\right)$; it follows that $g\left(A_{f}\right)-g\left(\left\{x_{n}\right\}_{n=1}^{\infty}\right)>l$. This contradicts the assumption that $x_{n} \stackrel{w}{\rightarrow} x, n \rightarrow \infty$.

Case 2. If $\left\{x_{n}\right\}_{n=1}^{\infty} \cap A_{f} \neq \emptyset$, then $\left(\left\{x_{n}\right\}_{n=1}^{\infty} \backslash A_{f}\right) \cap$ $A_{f}=\emptyset$. According to Case 1 we know that $\left\{x_{n}\right\}_{n=1}^{\infty} \backslash A_{f}$ is relatively compact set. Hence $\left\{x_{n}\right\}_{n=1}^{\infty} \cap A_{f}$ is compact set since $A_{f}$ is bounded closed convex set in certain finite dimensional subspace of $X$. On the other hand, it is obvious that $\left\{x_{n}\right\}_{n=1}^{\infty}=\left(\left\{x_{n}\right\}_{n=1}^{\infty} \backslash A_{f}\right) \cup\left(\left\{x_{n}\right\}_{n=1}^{\infty} \cap A_{f}\right)$; hence $\overline{\left\{x_{n}\right\}_{n=1}^{\infty}}=$ $\overline{\left(\left\{x_{n}\right\}_{n=1}^{\infty} \backslash A_{f}\right)} \cup \overline{\left(\left\{x_{n}\right\}_{n=1}^{\infty} \cap A_{f}\right)}$. This shows that $\left\{x_{n}\right\}_{n=1}^{\infty}$ is relatively compact.

Consequently, in Cases 1 and 2, we always conclude that $\left\{x_{n}\right\}_{n=1}^{\infty}$ is relatively compact. Furthermore, by the assumption that $x_{n} \stackrel{w}{\rightarrow} x, n \rightarrow \infty$, we can deduce that $\left\|x_{n}-x\right\| \rightarrow$ $0, n \rightarrow \infty$. This completes the proof that $X$ has the KadecKlee property.

In particular, considering the special case of Theorem 15 when $k=1$, we obtained Theorem 2.5 in [7] as a corollary.

Corollary 16. $X$ is uniformly extremely convex space if and only if $X$ is reflexive and for any $f \in S\left(X^{*}\right)$, one has $\operatorname{dim} A_{f}=$ $1, A_{f} \cap \overline{\mathrm{co}}\left(U(X) \backslash V_{A_{f}}\right)=\emptyset$, where the set $V_{A_{f}}$, which includes set $A_{f}$, is arbitrary open set with regard to norm topology $(X,\|\cdot\|)$. In other words, $X$ is uniformly extremely convex space if and only if $X$ is reflexive and every point of $S(X)$ is a denting point of $U(X)$.

To show that the converse to Theorem 8 is not true, we consider the following example.

Example 17. There exists a $k$-uniformly extremely convex space $X$ which is not a $(k-1)$-uniformly extremely convex space.

Let $k \geq 2$ be an integer, and let $i_{1}<i_{2}<\cdots<i_{k}$. For each $x=\left(a_{1}, a_{2}, \ldots\right) \in l_{2}$, define

$$
\|x\|_{i_{1}, \ldots, i_{k}}^{2}=\left(\sum_{j=1}^{k}\left|a_{i_{j}}\right|\right)^{2}+\sum_{i \neq i_{1}, \ldots, i_{k}} a_{i}^{2} .
$$

From [20] we know that $X_{i_{1}, \ldots, i_{k}}=\left(l_{2},\|\cdot\|_{i_{1}, \ldots, i_{k}}\right)$ is a $k \mathrm{UR}$ space. It is easy to see that $k \mathrm{UR}$ space is $k$-uniformly extremely convex space from the definition of $k U R$ space and a condition which characterizes $k$-uniformly extremely convex space in Lemma 9. Hence $X_{i_{1}, \ldots, i_{k}}$ is a $k$-uniformly extremely convex space. It follows from Theorem 11 that $X_{i_{1}, \ldots, i_{k}}$ is a $k$-strictly convex space but is not a $(k-1)$-strictly convex space that follows from [16]. Hence $X_{i_{1}, \ldots, i_{k}}$ is not a $(k-1)$-uniformly extremely convex space.

\section{The Relations between $k$-Uniformly Extremely Convex Space and Various Other Types of Convex Space}

Now we give a list of examples to distinguish $k$-uniformly extremely convex spaces from $k \mathrm{R}, k \mathrm{UR}, k \mathrm{NUC}$, and NUC spaces.

(i) We are ready now to distinguish $k$-uniformly extremely convex and $k \mathrm{R}$ spaces.

Since $k \mathrm{R}$ spaces are $k$-strictly convex spaces and have the drop property, it follows from Theorem 11 that $k \mathrm{R}$ spaces are $k$-uniformly extremely convex, but the converse is not true.

Example 18. There exists a $k$-uniformly extremely convex space $X$ which is not a $k \mathrm{R}$ space for every $k \geq 2$.

Let $k \geq 2$ be an integer, and let $i_{1}<i_{2}<\cdots<i_{k}$. For each $x=\left(a_{1}, a_{2}, \ldots\right) \in l_{2}$, define

$$
\|x\|_{i_{1}, \ldots, i_{k}}^{2}=\left(\sum_{j=1}^{k}\left|a_{i_{j}}\right|\right)^{2}+\sum_{i \neq i_{1}, \ldots, i_{k}} a_{i}^{2},
$$

and let $X_{i_{1}, \ldots, i_{k}}=\left(l_{2},\|\cdot\|_{i_{1}, \ldots, i_{k}}\right)$. For $x \in l_{2}$, let $\|x\|_{k}=$ $\sup _{i_{1}<i_{2}<\cdots<i_{k}}\|x\|_{i_{1}, \ldots, i_{k}}, X_{k}=\left(l_{2},\|x\|_{k}\right)$. It follows from [20] that $X_{k}$ is a $k \mathrm{UR}$ space but is not a $k \mathrm{R}$ space. Hence $X_{k}$ is a $k$ uniformly extremely convex space since $X$ is a $k U R$ space.

(ii) We are ready now to distinguish $k$-uniformly extremely convex and $k \mathrm{UR}$ spaces.

Example 19. For all $k \geq 1$, there exists a $k$-uniformly extremely convex space $X$ which is not a $k \mathrm{UR}$ space.

$$
\begin{aligned}
& \text { Let } E=\left(l_{2},\|\cdot\|\right) ; \text { for } x=\left(a_{1}, a_{2}, \ldots\right) \in E \text {, define } \\
& \qquad \begin{aligned}
\|x\|^{2}= & \left\{\left|a_{1}\right|+\left(a_{2}^{2}+a_{3}^{2}+\cdots\right)^{1 / 2}\right\}^{2} \\
& +\left\{\left(\frac{a_{2}}{2}\right)^{2}+\cdots+\left(\frac{a_{n}}{n}\right)^{2}+\cdots\right\}^{2} .
\end{aligned}
\end{aligned}
$$

It follows from [2] that $X=\left(\sum \bigoplus E\right)_{l_{2}}$ is a $2 \mathrm{R}$ space; furthermore, $X=\left(\sum \bigoplus E\right)_{l_{2}}$ is a $k$-uniformly extremely convex space but is not a $k$ UR space [20].

(iii) We are ready now to distinguish $k$-uniformly extremely convex and $\mathrm{L} k \mathrm{UR}$ spaces.

We consider a nonreflexive L $k$ UR space $X$. Then $X$ is not a $k$-uniformly extremely convex space since $X$ is not reflexive. On the other hand, we consider Example 19; then $X=\left(\sum \bigoplus E\right)_{l_{2}}$ is a $2 \mathrm{R}$ space and it follows that $X$ is a $k$ uniformly extremely convex space for all $k \geq 1$. But $X$ is not a LkUR space that follows from [21].

(iv) We are ready now to distinguish $k$-uniformly extremely convex spaces and NUC or $k$ NUC spaces.

Example 20. For all $k \geq 1$, there exists a $k$-uniformly extremely convex space $X$ which is neither a NUC nor a $k$ NUC space for all $k \geq 2$. 
Let $(X,\|\cdot\|)$ be the $l_{2}$-sum of $\left\{l_{n}, n \geq 2\right\}$; then $(X,\|\cdot\|)$ is a $2 \mathrm{R}$ space with normalized basis $\left\{e_{n}\right\}$. Define, $\forall x=$ $\sum_{n=1}^{\infty} a_{n} e_{n} \in X$

$$
\||x|\|=\left\{\left(\left|a_{1}\right|+\left\|\sum_{n=2}^{\infty} a_{n} e_{n}\right\|\right)^{2}+\sum_{n=2}^{\infty}\left(\frac{a_{n}}{n}\right)^{2}\right\}^{1 / 2} .
$$

By Theorem 4 in [9], we know that $(X,\||\cdot|\|)$ is a $2 \mathrm{R}$ space but is not a LNUC space. It follows that $X$ is a $k$-uniformly extremely convex space for all $k \geq 1$ but is neither a NUC nor a $k$ NUC space for all $k \geq 2$.

Remark 21. (i) The class of $k$-uniformly extremely convex spaces lies strictly between the classes of $k \mathrm{UR}$ spaces and the $k$-strongly convex spaces.

(ii) The class of $k$-uniformly extremely convex spaces lies strictly between the classes of $k \mathrm{R}$ spaces and the class of $k$ strongly convex spaces.

(iii) The class of $k$-uniformly extremely convex spaces has no inclusive relation with the class of $\mathrm{L} k \mathrm{UR}$ spaces.

In particular, considering the special case of Remark 21 when $k=1$, we obtained the main conclusions of [7], that is, Remarks 3.5 and 3.7 in [7].

\section{Competing Interests}

The authors declare that they have no competing interests.

\section{Acknowledgments}

This work was supported by the National Natural Science Foundation of China (Grant no. 11561053).

\section{References}

[1] J. A. Clarkson, "Uniformly convex spaces," Transactions of the American Mathematical Society, vol. 40, no. 3, pp. 396-414, 1936.

[2] K. Fan and I. Glicksberg, "Fully convex normed linear spaces," Proceedings of the National Academy of Sciences of the United States of America, vol. 41, pp. 683-698, 1955.

[3] R. E. Huff, "Banach spaces which are nearly uniformly convex," The Rocky Mountain Journal of Mathematics, vol. 10, no. 4, pp. 743-749, 1980.

[4] D. N. Kutzarova, " $k-\beta$ and k-Nearly uniformly convex Banach spaces," Journal of Mathematical Analysis and Applications, vol. 162, no. 2, pp. 322-338, 1991.

[5] S. Q. Shang, Y. A. Cui, and Y. Q. Fu, "Nearly dentability and approximative compactness and continuity of metric projector in Banach spaces," Scientia Sinica Mathematica, vol. 41, no. 9, pp. 815-825, 2011 (Chinese).

[6] F. Sullivan, "A generalization of uniformly rotund Banach spaces," Canadian Journal of Mathematics, vol. 31, no. 3, pp. 628636, 1979.

[7] S. Wulede and W. Ha, "A new class of Banach space with the drop property," Proceedings of the Royal Society of EdinburghSection A: Mathematics, vol. 142, no. 1, pp. 215-224, 2012.

[8] K. Fan and I. Glicksberg, "Some geometric properties of the spheres in a normed linear space," Duke Mathematical Journal, vol. 25 , pp. $553-568,1958$.
[9] D. N. Kutzarova and B.-L. Lin, "Locally $k$-Nearly uniformly convex Banach spaces," Mathematica Balkanica, vol. 8, no. 2-3, pp. 203-210, 1994.

[10] I. Singer, "On the set of best approximation of an element in a normed linear space," Revue Roumaine des Mathematiques Pures et Appliquees, vol. 5, pp. 383-402, 1960.

[11] C. X. Wu and Y. J. Li, "Strong convexity in Banach spaces," Journal of Mathematics, vol. 13, no. 1, pp. 105-108, 1993, (Chinese).

[12] S. Wulede and C. X. Wu, " $K$-strong convexity and $K$-strong smoothness," Chinese Annals of Mathematics A, vol. 19, no. 3, pp. 373-378, 1998 (Chinese).

[13] S. Wulede, " $k$-super-strongly convex and $k$-super-strongly smooth Banach spaces," Journal of Mathematical Analysis and Applications, vol. 298, no. 1, pp. 45-56, 2004.

[14] S. Rolewicz, "On drop property," Studia Mathematica, vol. 85, pp. 27-35, 1987.

[15] V. Montesinos, "Drop property equals reflexivity," Studia Mathematica, vol. 87, no. 1, pp. 93-100, 1987.

[16] C. X. Nan and J. H. Wang, " $k$-strict convexity and $k$ smoothness," Chinese Annals of Mathematics A, vol. 11, no. 3, pp. 321-324, 1990 (Chinese).

[17] Z. H. Zhang and X. N. Fang, "Characterizations of the $k$ strong convexity," Acta Scientiarum Naturalium Universitatis Nankaiensis, vol. 33, no. 4, pp. 100-104, 2000 (Chinese).

[18] M. M. Day, Normed Linear Spaces, Springer, New York, NY, USA, 3rd edition, 1973.

[19] A. R. Lovaglia, "Locally uniformly convex Banach spaces," Transactions of the American Mathematical Society, vol. 78, pp. 225-238, 1955.

[20] B.-L. Lin and X. T. Yu, "On the $k$-uniform rotund and the fully convex Banach spaces," Journal of Mathematical Analysis and Applications, vol. 110, no. 2, pp. 407-410, 1985.

[21] C. X. Nan and J. H. Wang, "On the LkUR and LkR spaces," Mathematical Proceedings of the Cambridge Philosophical Society, vol. 104, no. 3, pp. 521-526, 1988. 


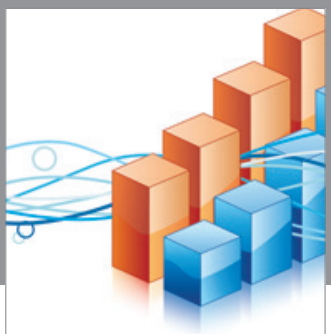

Advances in

Operations Research

vatem alat4

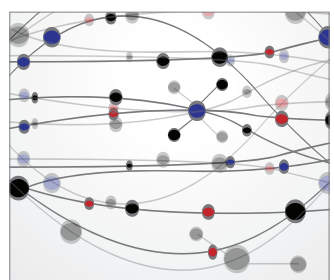

\section{The Scientific} World Journal
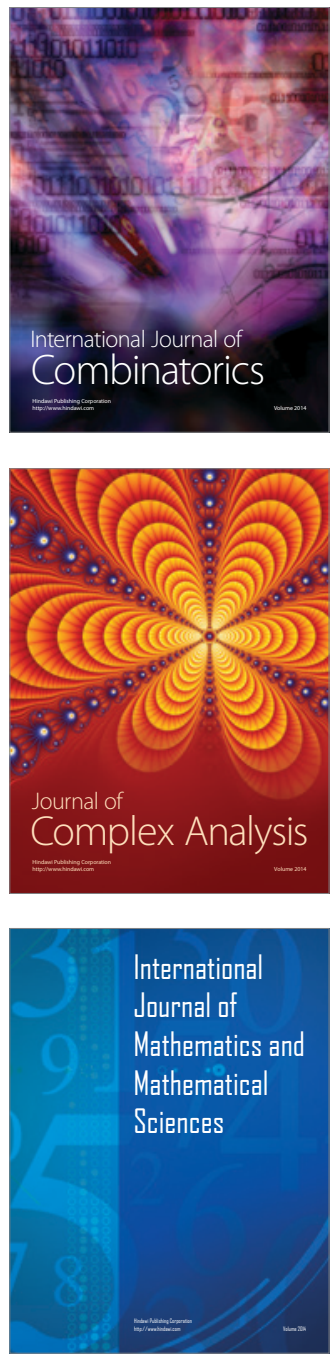
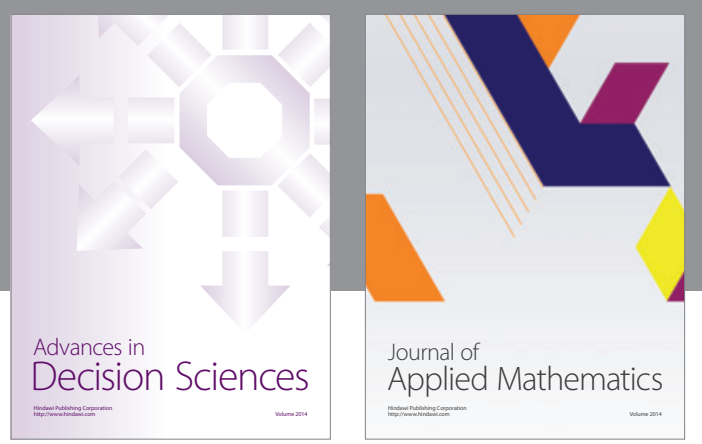

Algebra

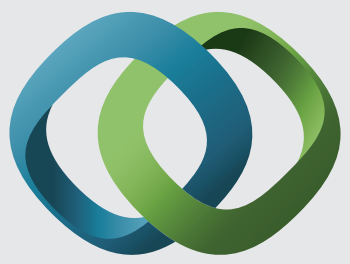

\section{Hindawi}

Submit your manuscripts at

http://www.hindawi.com
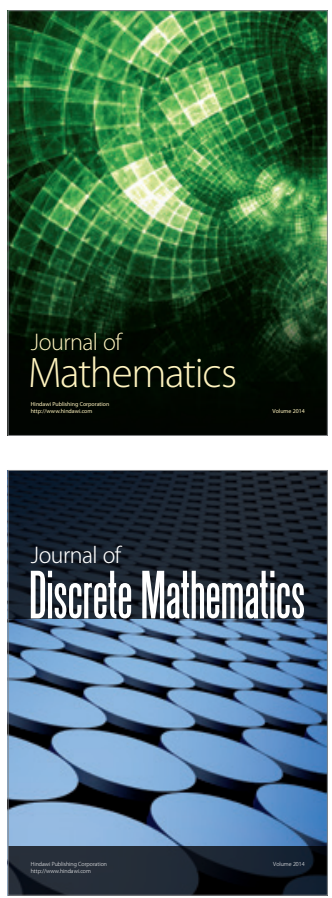

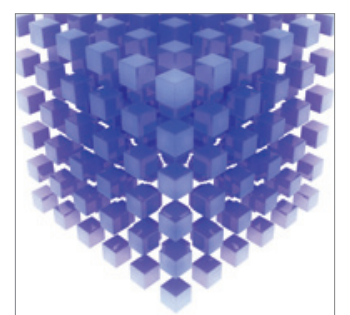

Mathematical Problems in Engineering
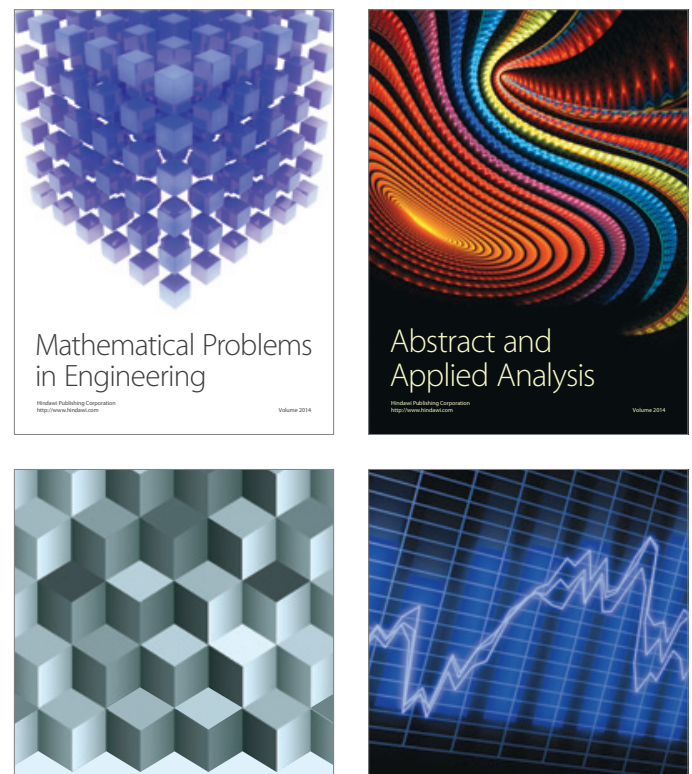

Journal of

Function Spaces

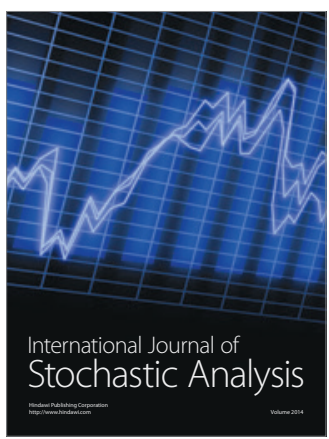

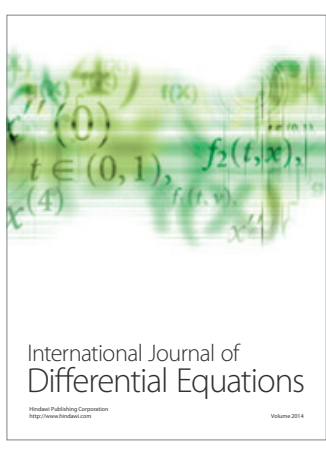
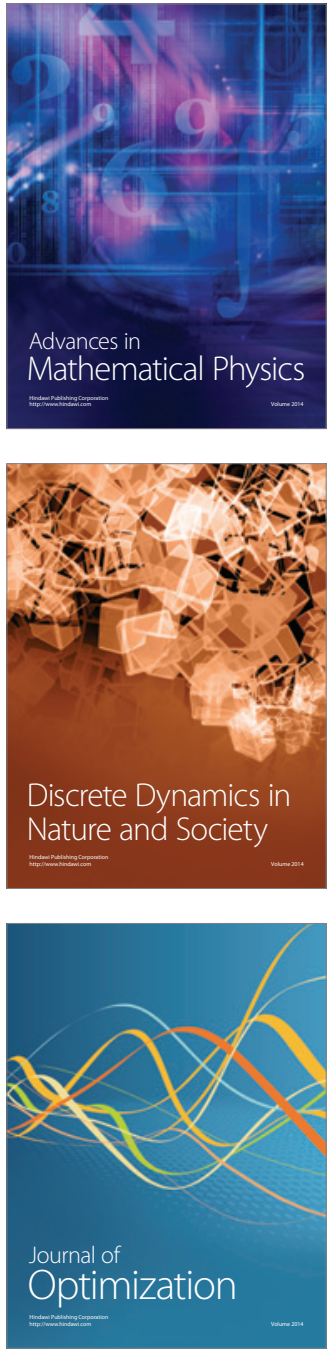